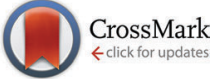

Cite this: Soft Matter, 2015, 11,4180

Received 22nd October 2014, Accepted 16th March 2015

DOI: $10.1039 / \mathrm{c} 4 \mathrm{sm} 02325 \mathrm{k}$

www.rsc.org/softmatter

\title{
A novel method to determine the elastic modulus of extremely soft materials
}

\author{
Tamás Stirling $^{\mathrm{a}}$ and Miklós Zrínyi ${ }^{\mathrm{ab}}$
}

\begin{abstract}
Determination of the elastic moduli of extremely soft materials that may deform under their own weight is a rather difficult experimental task. A new method has been elaborated by means of which the elastic modulus of such materials can be determined. This method is generally applicable to all soft materials with purely neo-Hookean elastic deformation behaviour with elastic moduli lower than $1 \mathrm{kPa}$. Our novel method utilises the self-deformation of pendent gel cylinders under gravity. When suspended, the material at the very top bears the weight of the entire gel cylinder, but that at the bottom carries no load at all. Due to the non-uniform stress distribution along the gel sample both the stress and the resulting strain show position dependence. The cross-sectional area of the material is lowest at the top of the sample and gradually increases towards its bottom. The equilibrium geometry of the pendant gel is used to evaluate the elastic modulus. Experimental data obtained by the proposed new method were compared to the results obtained from underwater measurements. The parameters affecting the measurement uncertainty were studied by a Pareto analysis of a series of adaptive Monte Carlo simulations. It has been shown that our method provides an easily achievable method to provide an accurate determination of the elastic modulus of extremely soft matter typically applicable for moduli below $1 \mathrm{kPa}$.
\end{abstract}

\section{Introduction}

The elastic modulus of materials is an important characteristic of their mechanical behaviour. The conventional method of determination is usually based on unidirectional elongation or compression. In the case of soft materials it is difficult to perform reliable measurements. The effect of deformation under the specimen's own weight can be decreased by measuring the elastic modulus in a liquid having the same density as that of the material. However, finding an appropriate liquid, which has the same density but does not react with the material and does not alter its mechanical properties, can sometimes be both challenging and expensive, especially in the case of complex materials. The other technical difficulty arises from gripping the end of a soft sample between the specimen clips during elongation measurements, which results in inhomogeneous deformation. At unidirectional compression the barrel distortion may cause experimental error. Due to these phenomena measurements based on unidirectional stress-strain dependence are difficult to evaluate. Although alternative methods do exist, such as the bending cylinder under gravity

\footnotetext{
${ }^{a}$ Molecular Biophysics Research Group, Hungarian Academy of Sciences,

Nagyvarad sq 4, H-1089 Budapest, Hungary

${ }^{b}$ Laboratory of Nanochemistry, Department of Biophysics and Radiation Biology,

Semmelweis University, Nagyvarad sq 4, H-1089 Budapest, Hungary
}

by Peng et al., ${ }^{1}$ these methods are only feasible for materials that are still harder than those discussed herein. The main motivation of the present work was to develop a method which exploits the deformation of the suspended cylindrical samples under their own weight. In this work we have studied the unidirectional stress-strain properties of extremely soft materials, i.e. materials that have moduli below $1 \mathrm{kPa}$.

\section{Theoretical background}

On the basis of the rubber elasticity theory, ${ }^{2-5}$ it is possible to express the elastic free energy density as a function of the principal deformation ratios $\lambda_{x}, \lambda_{y}$ and $\lambda_{z}$. These quantities are defined as the ratio of deformed and undeformed dimensions corresponding to the directions $x, y$ and $z$, respectively.

$$
a_{\mathrm{el}}=a_{0}+\frac{1}{2} \cdot G \cdot\left(\lambda_{x}^{2}+\lambda_{y}{ }^{2}+\lambda_{z}{ }^{2}-3\right)
$$

where $a_{\mathrm{el}}$ represents the elastic part of the free energy density, $G$ means the elastic modulus and $a_{0}$ stands for the free energy density of the undeformed gel sample $\left(\lambda_{x}=\lambda_{y}=\lambda_{z}=1\right)$. As the volume of the sample remains constant during deformation, the product of the principal deformations equals one.

$$
\lambda_{x} \cdot \lambda_{y} \cdot \lambda_{z}=1
$$

It is known that in general the elastic properties of gels cannot be satisfactorily described by the Gaussian network models. 
Another widely used model for the description of such materials is the Mooney-Rivlin model. The free energy density function of an incompressible Mooney-Rivlin material is:

$$
W=C_{1} \cdot\left(I_{1}-3\right)+C_{2} \cdot\left(I_{2}-3\right)
$$

where $I_{1}$ and $I_{2}$ are the first and second strain invariants:

$$
\begin{gathered}
I_{1}=\lambda_{x}{ }^{2}+\lambda_{y}{ }^{2}+\lambda_{z}{ }^{2} \\
I_{2}=\lambda_{x}{ }^{2} \cdot \lambda_{y}{ }^{2}+\lambda_{x}{ }^{2} \cdot \lambda_{z}{ }^{2}+\lambda_{y}{ }^{2} \cdot \lambda_{z}{ }^{2}
\end{gathered}
$$

However, there is much experimental evidence showing that if the swelling degree exceeds a certain value, a virtual idealization of mechanical properties occur and $C_{2}$ can practically be considered as zero. ${ }^{6-8}$ This means that for highly swollen networks the Gaussian model can provide an adequate approximation.

The stress-strain dependence of a unidirectional deformed gel sample may be expressed on the basis of eqn (1) and (2). Let us consider a cylindrical gel extended along its axis $z$, so that the circular symmetry is maintained. Let $h_{0}$ and $h$ be the initial (reference) and the deformed length of the cylinder, respectively. Then the principal deformation ratio along the axis $z$ is $\lambda_{z}=h / h_{0}$ and $\lambda_{x}=\lambda_{y}=\sqrt{1 / \lambda_{z}}$ for all other directions perpendicular to the $z$ axis. Therefore eqn (1) can be rewritten as:

$$
a\left(\lambda_{z}\right)=a_{0}+\frac{1}{2} \cdot G \cdot\left(\lambda_{z}^{2}+2 \cdot \lambda_{z}^{-1}-3\right)
$$

The stress can be derived from eqn (6) using a standard method. ${ }^{2-5}$ The result is called the neo-Hookean law:

$$
\sigma_{\mathrm{N}}=G \cdot\left(\lambda_{z}-\lambda_{z}{ }^{-2}\right)
$$

where the nominal stress, $\sigma_{\mathrm{N}}$, is defined as the ratio of the equilibrium elastic force and the undeformed cross-sectional area of the sample.

The neo-Hookean law described in eqn (7) is generally applicable in most cases, but the suspension of a sample made of an extremely soft material results in more complex geometries due to the inhomogeneity of stress distribution across the length of the sample (see Fig. 2). In order to describe the spatial dependence of the equilibrium cross-sectional area in a suspended sample we propose a model, which assumes that the sample is deformed into a series of cylinders or prisms as shown in Fig. 1.

The gel cylinder at the very top bears the largest weight, therefore this part is the longest and the narrowest. Each proceeding cylinder bears less weight and is therefore shorter but wider than the previous. At the bottom, the gel piece carries no load at all, therefore it undergoes no deformation. In order to give a better estimation of the elastic modulus it was thought beneficial to use a more generalized form of the neo-Hookean law, which utilizes more experimental data. Therefore, we included not only the elongation, but also the contraction of a given segment of the gel. The neo-Hookean law can thus be written in the following form:

$$
\sigma_{\mathrm{R}}(n)=G \cdot\left[\lambda_{\mathrm{e}}^{2}(n)-\lambda_{\mathrm{r}}^{2}(n)\right]
$$

where $n$ denotes the sequential number of each sample segment, starting from the top of the sample, $\sigma_{\mathrm{R}}$ stands for the real stress,

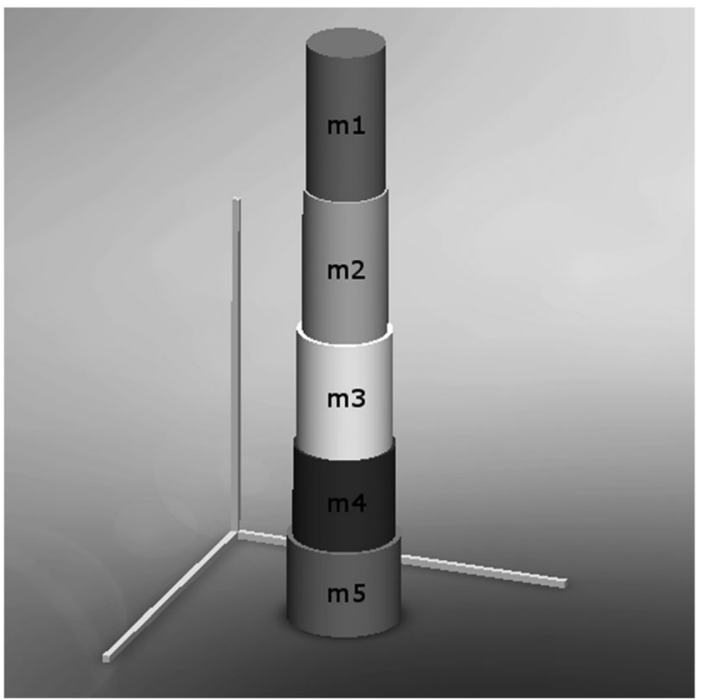

Fig. 1 Concept of the model. A cylindrical sample is deformed into a series of cylinders as a result of suspension, each with its own length, diameter and weight.

$\lambda_{\mathrm{e}}$ is the vertical deformation ratio defined as the ratio of the suspended and unsuspended length of a segment, and $\lambda_{\mathrm{r}}$ means the horizontal deformation ratio of suspended and unsuspended diameters of a gel segment.

Eqn (8) can be derived from eqn (7) by taking into consideration the conservation of volumes $\left(\lambda_{x} \cdot \lambda_{y} \cdot \lambda_{z}=\lambda_{\mathrm{e}} \cdot \lambda_{\mathrm{r}}{ }^{2}=1\right)$ as well as the general relationship between nominal and real stresses.

$$
\begin{aligned}
\sigma_{\mathrm{N}}(n) & =\frac{F(n)}{A_{0}}=\frac{F(n)}{A(n)} \cdot \frac{A(n)}{A_{0}}=\sigma_{\mathrm{R}}(n) \cdot \frac{d^{2} \cdot \pi / 4}{d_{0}^{2} \cdot \pi / 4} \\
& =\sigma_{\mathrm{R}}(n) \cdot \lambda_{\mathrm{r}}{ }^{2}(n)=\sigma_{\mathrm{R}}(n) \cdot \frac{1}{\lambda_{\mathrm{e}}(n)}
\end{aligned}
$$

where $F(n)$ is the force affecting the cross-sectional area, $A(n)$ is the cross-sectional area of the $n$-th part of the suspended gel piece and $A_{0}$ means the unsuspended cross-sectional area, $d$ and $d_{0}$ are the diameters of the corresponding cross-sections. It must be mentioned that, in the case of a suspended sample, the actual geometry between two adjacent marks is not really a cylinder, but more a conical frustum. In order to facilitate modelling, each conical frustum was mathematically transformed into a hypothetical cylinder with the same height and volume as the corresponding conical frustum, and a diameter, $d_{\text {calc }}$. This diameter was calculated from the equality of volumes:

$$
d_{\mathrm{calc}}=\sqrt{\frac{d^{2}+d \cdot D+D^{2}}{3}}
$$

where $d_{\text {calc }}$ is the equivalent diameter of the hypothetical cylinder, and $d$ and $D$ are the measured upper and lower diameters of the conical frustum, respectively.

In the proposed model - despite the continuous spatial dependence of stresses and deformations - the real stress decreases step-by-step from the top of the specimen towards its bottom, in accordance with the amount of load under each 
segment. This latter is considered to be constant within each individual segment.

The only relevant measurable quantity related to the forces affecting a sample segment is the total weight of the sample. According to the presented model, each segment is affected by the total weight of segments below it but not by the segment in question and not by those above it. Therefore, if the sample is divided into " $n$ " number of segments, then the first segment is affected by the weight of the other " $n-1$ " segments, the second by the other " $n-2$ " and so on. The last segment is not affected by any other and thus remains undeformed. As the density of the sample was assumed to be constant during deformation, the weight affecting a certain segment was considered to be proportional to its volume, and was calculated accordingly. In order to calculate $\sigma_{\mathrm{R}}$, weights were multiplied by the gravitational acceleration and were divided by the crosssectional area of the segment, which was calculated from the equivalent diameter of each hypothetical cylinder. As $\sigma_{\mathrm{R}}$ refers to real stresses, and not nominal stresses, the required crosssectional areas were those of the suspended sample and those of the unsuspended.

According to the model, if the position dependent $\sigma_{\mathrm{R}}, \lambda_{\mathrm{e}}$ and $\lambda_{\mathrm{r}}$ data can be determined via measurement of each sample segment, then plotting $\sigma_{\mathrm{R}}$ against $\left[\lambda_{\mathrm{e}}{ }^{2}(n)-\lambda_{\mathrm{r}}{ }^{2}(n)\right]$ yields a linear curve, and the slope provides the shear modulus of the sample. This is only true however as long as $G$ is constant, i.e. for small to moderate deformations and constant temperature.

\section{Experimental}

\section{Sample preparation}

For the experiments, gel systems of polyvinyl alcohol (PVA) swollen to equilibrium in water were used. The samples were prepared from high molecular weight polyvinyl alcohol ( $M=124000-186000 \mathrm{~g} \mathrm{~mol}^{-1}$, Aldrich) cross-linked with glutaraldehyde (Merck) in water under acidic conditions $(\mathrm{pH} \approx 2)$. The PVA concentration of these hydrogels was $4.5 \mathrm{wt} \%$ during preparation and their crosslink density (CD), which is the molar ratio of monomer units to crosslinkers in the system, ranged between 300 and 400 . The polymer solution, the crosslinking agent, and the catalyst $(1: 1 \mathrm{HCl}, \mathrm{VWR})$ were manually mixed in beakers and were afterwards poured into cylindrical containers. After the crosslinking reaction took place the gels were removed from the containers and were put in distilled water. The water was then removed and changed several times in order to get rid of remnants of unreacted materials. Before elastic measurements, the gel cylinders were removed from water, and while unsuspended, they were marked with paint throughout their length at quasi-equidistant intervals (approx. $1 \mathrm{~cm}$ ).

\section{Determination of the elastic modulus by the pendant gel method}

Samples were glued to a cork at their upper ends, afterwards they were marked at quasi-equidistant intervals and then they were suspended using a extensometer as shown in Fig. 2. The

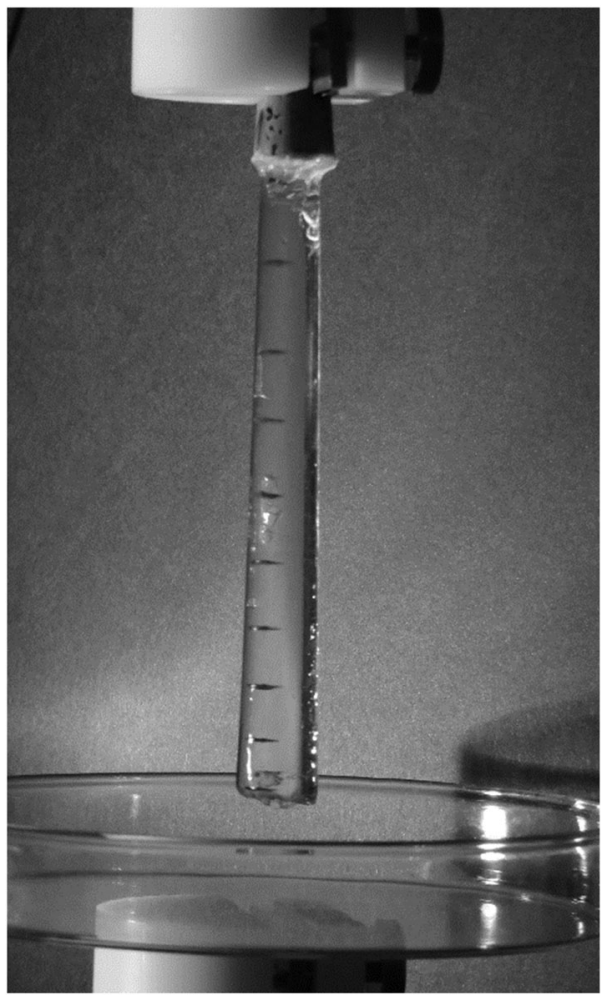

Fig. 2 A suspended cylindrical sample. Unsuspended length was approx. $100 \mathrm{~mm}$, suspended length was approx. $125 \mathrm{~mm}$. Crosslink density was 350 .

extensometer allowed for smooth lifting of the samples which was very important especially in the case of the softest samples, as it prevented them from tearing at the top, where internal stresses were close to the tensile strength of the materials. Photographs were taken of both the unsuspended and the suspended samples. All photographs were individually calibrated using a digital caliper. The picture resolution was initially 10 pix per $\mathrm{mm}$, but was later increased to 100 pix per $\mathrm{mm}$. The accuracy of distance measurements was estimated to be \pm 5 pix. During evaluation of the photographs sample diameters were measured at each mark along with the distances of adjacent marks.

To give the most precise estimation of the sample weight at the time of suspension, the weight of the samples was measured both before and after suspension along with the exact times of each weight measurement. As the exact time of the photograph taken in suspension was also recorded, the exact weight of the sample in suspension could be estimated by linear interpolation. Although the weight reduction during the measurement was usually only a few percent (around 3-5\% in most cases, probably due to evaporation), all stresses were calculated from sample weights, therefore it was thought beneficial to determine sample weights as accurately as possible. The accuracy of the analytical balance, provided by its manufacturer, was $0.0001 \mathrm{~g}$.

\section{Unidirectional extension measurements}

If an extremely soft gel sample is put into an environment which has a density similar or equal to its own, then the 
extension due to its own weight will be reduced, and the modulus of the material can be measured by stress-strain measurement in an extensometer. For our hydrogel samples we used distilled water. Such an experiment is shown in Fig. 3.

Due to the extreme softness of our materials, it was impossible to keep the ends of the samples within the specimen clips of the extensometer. Therefore, samples were glued to corks which were cut at their opposite sides to a proper size, and it was the corks which were gripped by the specimen clips of the extensometer. The instrument used in these measurements was an Instron 5942 extensometer ( $0.5 \mathrm{kN}$ capacity, $726 \mathrm{~mm}$ vertical test space).

\section{Results and discussion}

\section{Determination of the elastic modulus by the pendant gel method}

In order to implement the concept presented in Fig. 1 and formulated by eqn (8), $\lambda_{\mathrm{e}}, \lambda_{\mathrm{r}}$, and $\sigma_{\mathrm{R}}$ must be determined properly for all the $n$ segments of the sample.

The distances between two adjacent marks and the diameters at each mark were measured for all samples and the equivalent diameters were calculated by eqn (10) for each sample segment, both suspended and unsuspended. $\lambda_{\mathrm{e}}$ is the ratio of suspended and unsuspended distances, and $\lambda_{\mathrm{r}}$ is the ratio of the suspended and unsuspended equivalent diameters.

Experimental data of $\lambda_{\mathrm{e}}$ and $\lambda_{\mathrm{r}}$ values of succeeding sample segments are presented in Fig. 4-6 for three hydrogels with three different crosslink densities, 300, 350 and 400, respectively. It can be seen that while the deformation of most

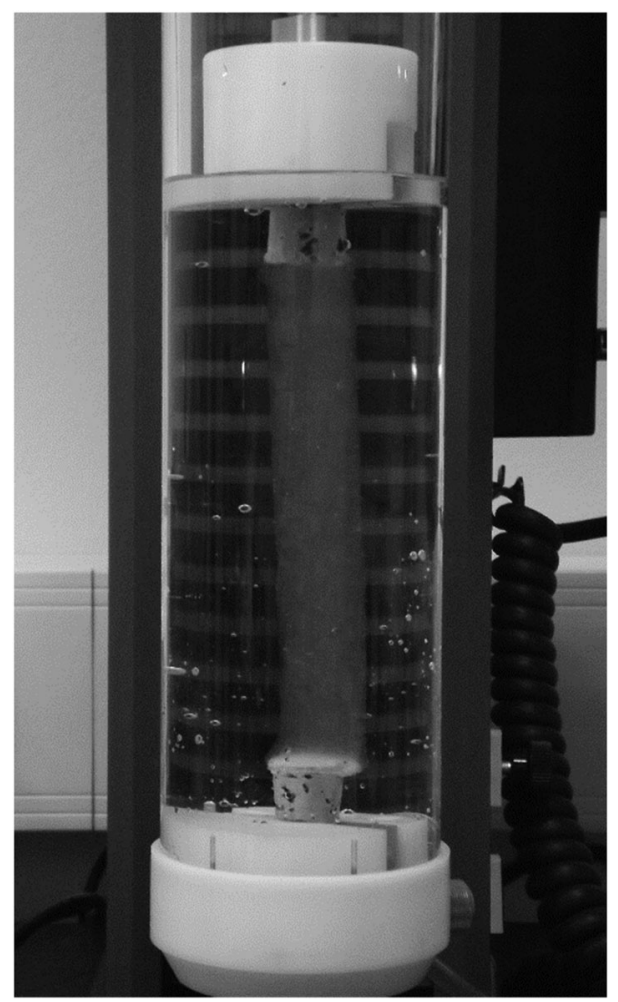

Fig. 3 Stress-strain measurements of a hydrogel in water. segments changes according to the model, i.e. each vertical deformation ratio is lower and each horizontal deformation ratio is higher than that of the preceding sample, those of the first two and the last sample segments are different from which was anticipated and cannot be justified by the proposed model. Deviations at the top of the samples are probably due to the gluing required to be able to suspend the samples, while those at the very bottom are due to the difficulty in measuring the geometries of complex shapes and the priority of properly estimating the volume of the last sample segment (which is required for all stress calculations) compared with the precise measurement of the elongation and radial contraction of an individual sample segment. Although these segments were also involved in all calculations, they produced outliers in most cases, and were omitted from the plots which were used to determine the modulus.

Experimental data were analysed on the basis of eqn (8). As the result of an ordinary least squares regression is correct only if the underlying assumptions are true, and is sensitive to the violation of its assumptions (e.g. homoscedasticity, independency

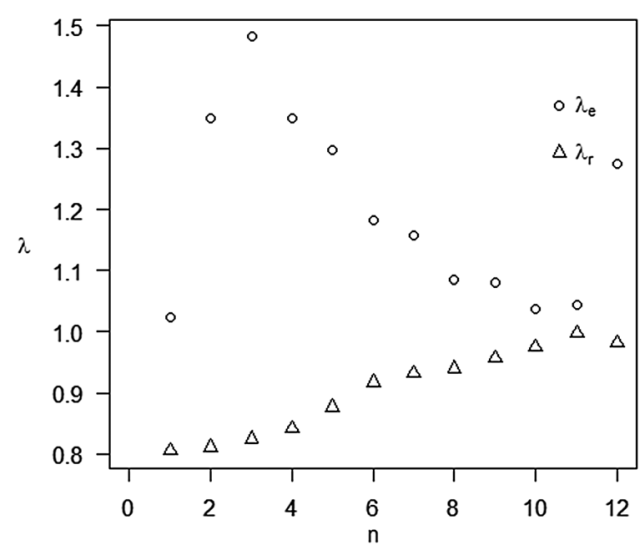

Fig. 4 Elongation and radial contraction of each sample segment in a sample (CD_300_2). $n$ is the sequential number of each sample segment from the top to the bottom of the sample.

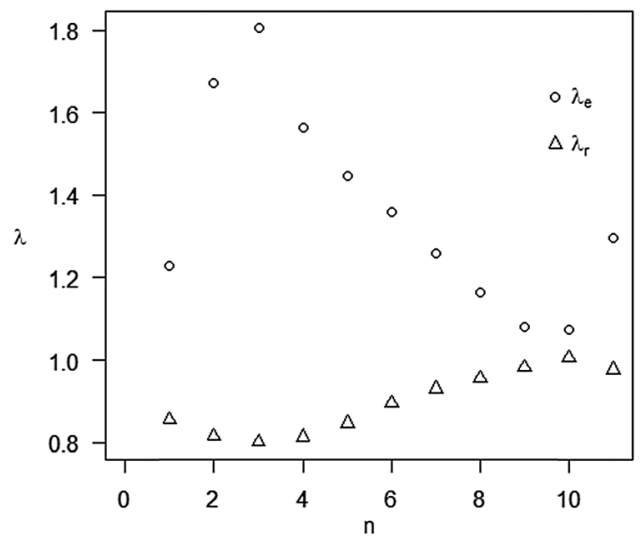

Fig. 5 Elongation and radial contraction of each sample segment in a sample (CD_350_2). $n$ is the sequential number of each sample segment from the top to the bottom of the sample. 


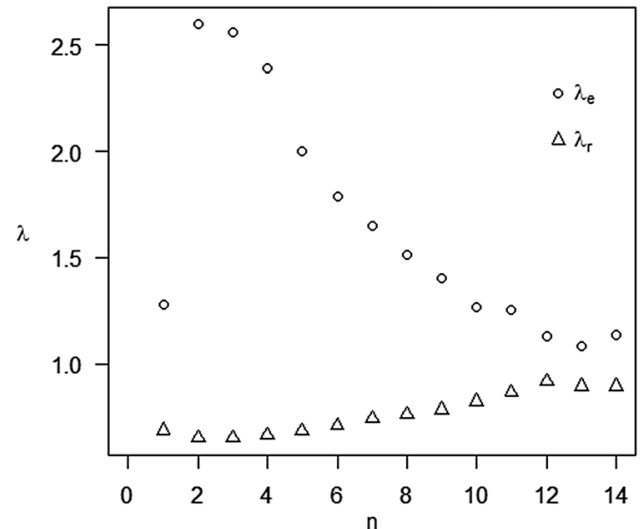

Fig. 6 Elongation and radial contraction of each sample segment in a sample (CD_400_1). $n$ is the sequential number of each sample segment from the top to the bottom of the sample.

and normal distribution of errors) and especially sensitive to outliers, it was thought more convenient to apply a robust linear regression model to evaluate experimental results.

To visualize the goodness of fit, the modulus calculated as the slope of this linear curve was used to simulate the location of each mark on the suspended sample from that of the
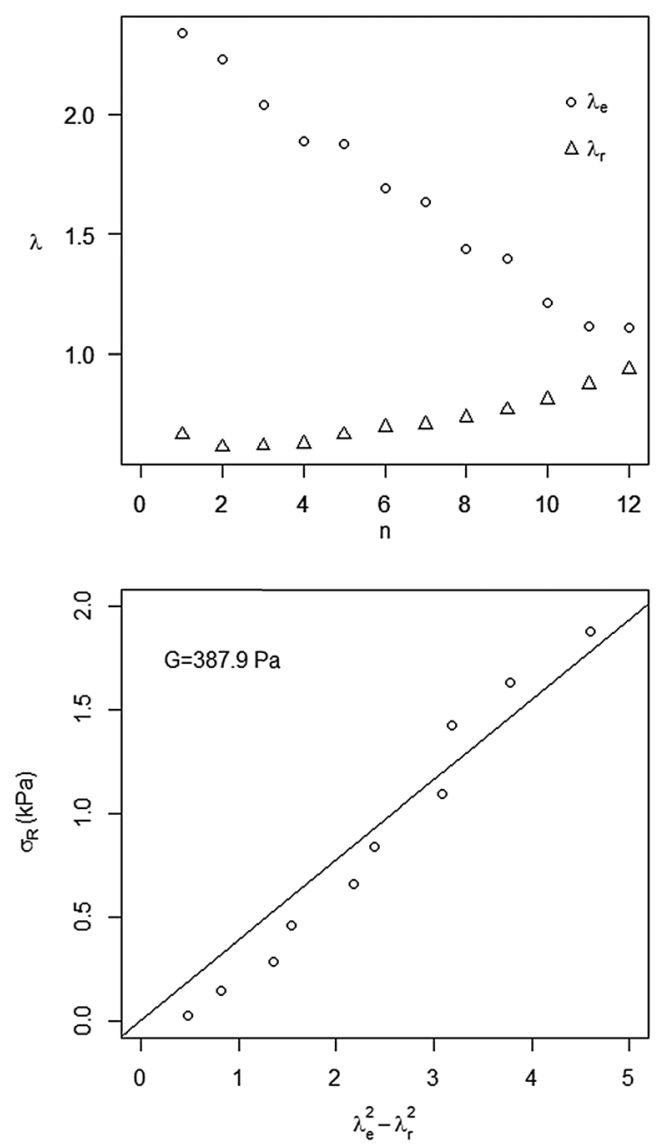

Fig. 7 Elongation, radial contraction and stress-strain relationship of an approx. $100 \mathrm{~mm}$ long sample (CD = 350). Shear modulus was calculated according to the described model.

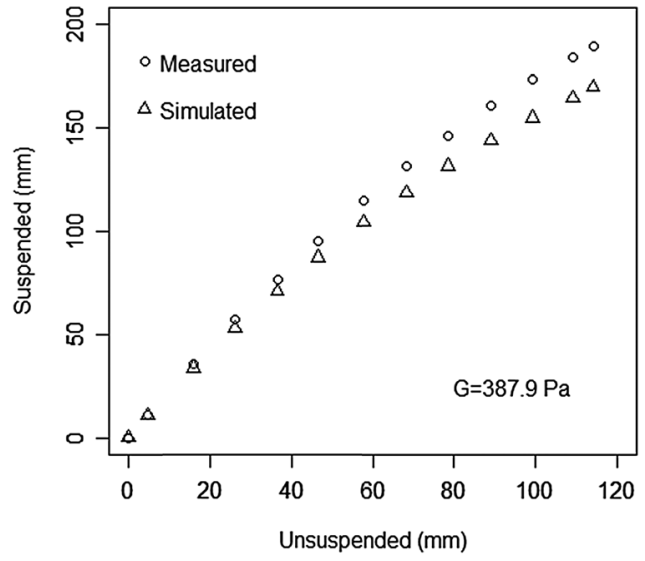

Fig. 8 Location of each mark on the unsuspended and suspended sample. Measured and simulated results.

unsuspended, by applying the neo-Hookean law to each sample segment. The results are shown in Fig. 8.

As can be seen in Fig. 8 the linear regression did not produce a good fit, and it seems like the real modulus of the sample is lower than the estimate. The difference was thought to be associated with the sensitivity of the model to measurement uncertainties and the general applicability of the linear model to this problem.

There are various reasons for the low precision of the model. Following a bottom-up approach, the first thing to consider is the uncertainty associated with measurement results. The theory and calculations presented herein are in accordance with the relevant international standards. ${ }^{9,10}$ Measurements in general have imperfections that give rise to errors. These errors can be systematic or random in nature. Systematic errors or 'biases' are errors which have a certain quantifiable effect on all measurement results. If a recognized effect on a measurement result can be quantified and proves to be significant in size relative to the required accuracy of the measurement, a correction can be applied to compensate for the effect. Random errors on the other hand are unpredictable and arise from stochastic processes which give rise to variations in repeated observations of the measurand. If a general quantity " $F$ " to be determined is a function of parameters " $x_{1}$ ", " $x_{2}$ ", $\ldots$, " $x_{n}$ ", then the uncertainty of " $F$ " could be estimated by a Pythagorean addition:

$$
\sigma_{F}{ }^{2}=\left(\frac{\delta F}{\delta x_{1}}\right)^{2} \cdot{\sigma_{x_{1}}}^{2}+\left(\frac{\delta F}{\delta x_{2}}\right)^{2} \cdot{\sigma_{x_{2}}}^{2}+\ldots+\left(\frac{\delta F}{\delta x_{n}}\right)^{2} \cdot{\sigma_{x_{n}}}^{2}
$$

In our experiments the quantity " $F$ " to be studied is the modulus and its uncertainty arises from the uncertainty of weight and distance measurements. According to the GUM, 9,10 the uncertainty of a weight measurement can be approximated by uniform distribution from the accuracy of the analytical balance, if it is provided by the manufacturer. Similarly, the uncertainty of distances measured by the caliper can also be estimated by uniform distribution from the accuracy provided by its manufacturer. Regarding the photographs it was assumed that all distances can be measured with the same \pm 5 pix accuracy, and the 
Table 1 Sources of uncertainty in our measurements

\begin{tabular}{lllll}
\hline Measurand & Symbol & Accuracy $( \pm)$ & Distribution & Uncertainty \\
\hline Weight & $\sigma_{\mathrm{m}}{ }^{2}$ & $0.0001(\mathrm{~g})$ & Uniform & $\sigma_{\mathrm{m}}^{2}=\frac{0.0001^{2}}{3}(\mathrm{~g})$ \\
Distance (caliper) & $\sigma_{l(\mathrm{~mm})}{ }^{2}$ & $0.02(\mathrm{~mm})$ & Uniform & $\sigma_{l(\mathrm{~mm})}^{2}=\frac{0.02^{2}}{3}(\mathrm{~mm})$ \\
Distance (photograph) & $\sigma_{l(\text { pix })}{ }^{2}$ & $5($ pix $)$ & Triangular & $\sigma_{l(\mathrm{pix})}^{2}=\frac{5^{2}}{6}($ pix $)$
\end{tabular}

uncertainty of a distance measurement can be approximated by triangular distribution, which is the distribution of the sum of two uniformly distributed variables. The various sources of uncertainties and their estimated values are summarized in Table 1.

According to eqn (11) and Table 1, the uncertainty of a photograph calibration can be estimated by the following equations:

$$
c=\frac{l(\mathrm{pix})}{l(\mathrm{~mm})}
$$

and

$$
\begin{aligned}
\sigma_{c}^{2} & =\left(\frac{\delta c}{\delta l(\mathrm{pix})}\right)^{2} \cdot \sigma_{l(\mathrm{pix})}{ }^{2}+\left(\frac{\delta c}{\delta l(\mathrm{~mm})}\right)^{2} \cdot \sigma_{l(\mathrm{~mm})}{ }^{2} \\
& \left.=\frac{1}{l(\mathrm{~mm})^{2}} \cdot\left(c^{2} \cdot \sigma_{l(\mathrm{~mm})^{2}}+\sigma_{l(\mathrm{pix})}\right)^{2}\right)
\end{aligned}
$$

where $c$ is the photograph resolution or the calibration ratio, $l$ (pix) is the calibration distance measured in pix and $l(\mathrm{~mm})$ is the same distance measured by the caliper in $\mathrm{mm}$. The same way the uncertainty of a distance measurement can be estimated from the following equations:

$$
l(\mathrm{~mm})=\frac{l(\mathrm{pix})}{c}
$$

and

$$
\begin{aligned}
\sigma_{l(\mathrm{~mm})}{ }^{2} & =\left(\frac{\delta l(\mathrm{~mm})}{\delta l(\mathrm{pix})}\right)^{2} \cdot \sigma_{l(\mathrm{pix})}{ }^{2}+\left(\frac{\delta l(\mathrm{~mm})}{\delta c}\right)^{2} \cdot{\sigma_{c}}^{2} \\
& =\frac{1}{c^{2}} \cdot\left(l(\mathrm{~mm})^{2} \cdot{\sigma_{c}}^{2}+\sigma_{l(\mathrm{pix})}{ }^{2}\right)
\end{aligned}
$$

where $l$ (pix) and $l(\mathrm{~mm})$ are not calibration distances anymore, but the actual distances on the sample to be measured.

Based on these considerations the adjustable parameters which contribute to the uncertainty of the modulus are the initial geometrical parameters of the sample (length, diameter and the number of sample segments) and the parameters of the calibration (calibration distance and calibration ratio). By assigning values to these quantities one can calculate an estimation of the modulus, and by altering these values one can screen for those effects which contribute the most to the estimated uncertainty.

Although the above equations provided proof for the para-

\begin{tabular}{|c|c|c|c|c|}
\hline & Symbol & Lower $(-)$ & Higher $(+)$ & Dimension \\
\hline Calibration distance & $l c$ & 10 & 50 & $\mathrm{~mm}$ \\
\hline Calibration ratio & $c$ & 10 & 100 & pix per mm \\
\hline Initial sample length & $h_{0}$ & 50 & 100 & $\mathrm{~mm}$ \\
\hline Initial sample diameter & $d_{0}$ & 10 & 20 & $\mathrm{~mm}$ \\
\hline Number of sample segments & $N$ & 5 & 10 & - \\
\hline
\end{tabular}
meters which come into consideration, the uncertainty of the modulus cannot be expressed in such a simple form but only by a numerical model, therefore the propagation of its uncertainty cannot be easily studied by the same method.
Table 2 Parameters altered during Monte Carlo trials

The general idea of a Monte Carlo propagation of uncertainty calculation is the repeated sampling from the probability distributions of each parameter and the calculation of the desired quantity from each sample taken. The result of the simulation will be a histogram of the desired quantity. The parameters altered in our simulations are presented in Table 2 .

For each set of parameters, suspended geometries were calculated from the unsuspended geometries by the neoHookean law, assuming a fixed $500 \mathrm{~Pa}$ modulus for the sample. This represented a situation when everything was measured precisely and there was no uncertainty associated with measurement results. Afterwards, random samples were taken around each calculated data assuming a given level of uncertainty (Table 1), and from the random samples the modulus was calculated by robust linear regression. The procedure involved an increasing number of Monte Carlo trials (adaptive Monte Carlo method), until the result of interest i.e. the standard deviation of the modulus has been stabilised, which usually required trials in the magnitude of $10^{5}$. Based on Table 2 a $2^{n}$ full factorial simulation experiment was designed, and the effect of each parameter on the 0.95 coverage interval of the modulus was investigated. The effects are summarized in a Pareto chart in Fig. 9.

Based on Fig. 9 the parameters responsible for the majority of uncertainties are the photograph resolution, or calibration ratio $c$, the calibration distance $l c$, and their interaction $l c: c$. A response surface was constructed to illustrate these effects (Fig. 10).

The response surface indicates that by improving the calibration i.e. increasing the resolution of the photographs and the calibration distance, the precision of the model can be significantly increased. This is shown in Fig. 11 and 12.

\section{Unidirectional extension measurements}

During the measurements the rod and the clips of the extensometer were also partially immersed in distilled water. These parts were made of materials that had different density than the density of the distilled water in which they were immersed, 


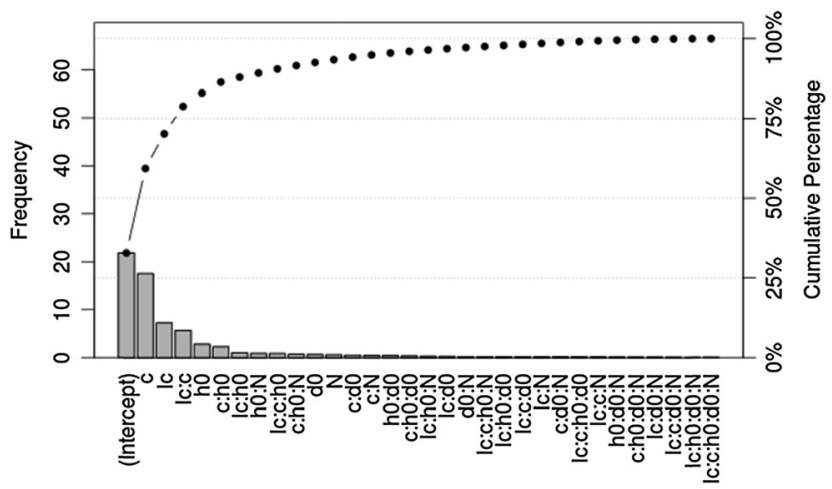

Fig. 9 Pareto chart of effects based on a $2^{n}$ full factorial experimental design of adaptive Monte Carlo simulations.

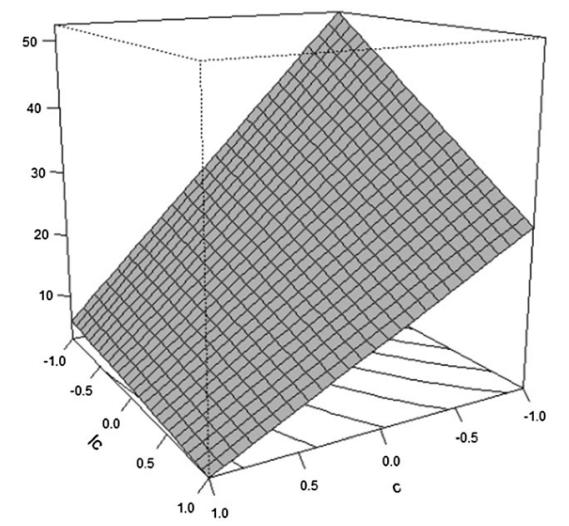

Fig. 10 Response surface of the effects of photograph resolution and the calibration distance on the 0.95 coverage interval of the modulus.

and were gradually removed from water during the course of the measurements, which resulted in time dependent buoyancy. Based on two consecutive measurements for each sample, the time dependent buoyancy was quantified and taken into correction.

The curve labelled "Sample" shows the classical unidirectional stress-strain relationship of a sample under distilled water. After the measurement, which lasted until $40 \mathrm{~mm}$ extension, the distilled water was removed from the container, the sample was cut off from the lower cork, leaving it hanging from the upper cork, then the distilled water was returned to the container, and the sample was returned to its initial position. Afterwards, the measurement was performed again, resulting in the curve labelled "Blind". The position of the dashed vertical line indicates a state of deformation where $\lambda_{\mathrm{e}}=1$. Data points to the right of the dashed line were omitted from further analysis. Results of the second measurement were then subtracted from those of the first, and the modulus was estimated as the slope of the resulting curve, using a cross-sectional area which was calculated from the average of the measured unsuspended diameters of the sample (Fig. 13).

\section{Improved evaluation of experimental data}

Fig. 7 and 11 show a linearization of the neo-Hookean law where the slope of the fitted linear curve is an estimation of the
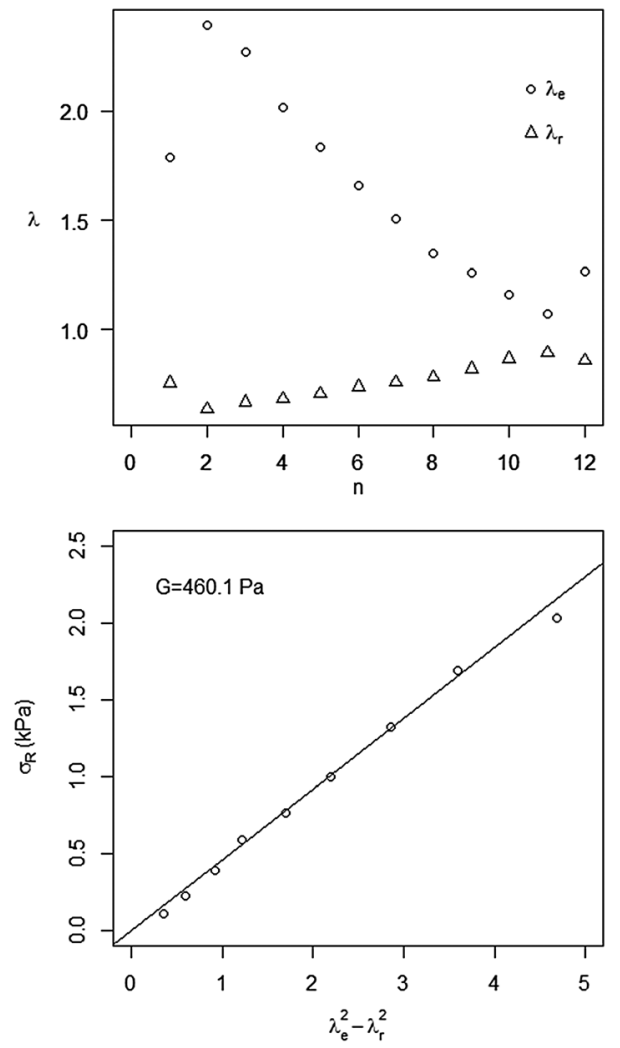

Fig. 11 Elongation, radial contraction and stress-strain relationship of an approx. $100 \mathrm{~mm}$ long sample $(C D \approx 350)$. Resolution was increased from 10 pix per $\mathrm{mm}$ to 100 pix per $\mathrm{mm}$.

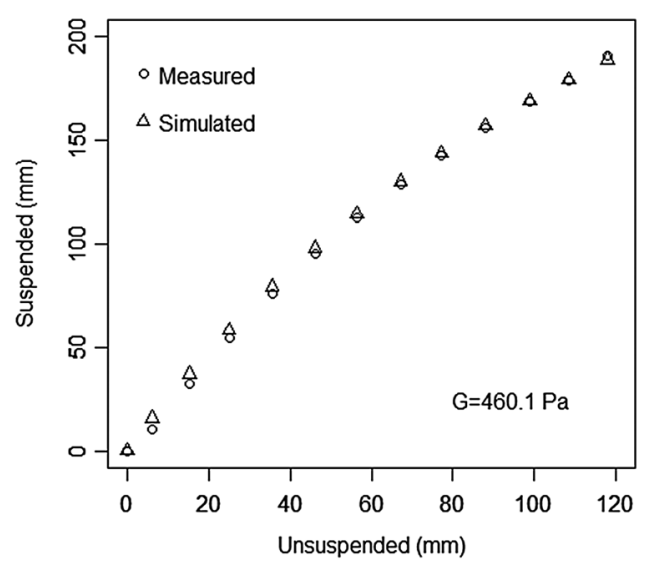

Fig. 12 Location of each mark on the unsuspended and suspended sample. Measured and simulated results.

shear modulus itself. However, due to the fact that the ordinate of the plot is derived from two weight measurements and a distance measurement, and the abscissa is derived from the second power of a series of individual distance measurements, it had to be verified that the assumptions of linear regression e.g., weak exogeneity (negligible variance of predictor variables), homoscedasticity (constant variance of the response variable), or the independence of errors 


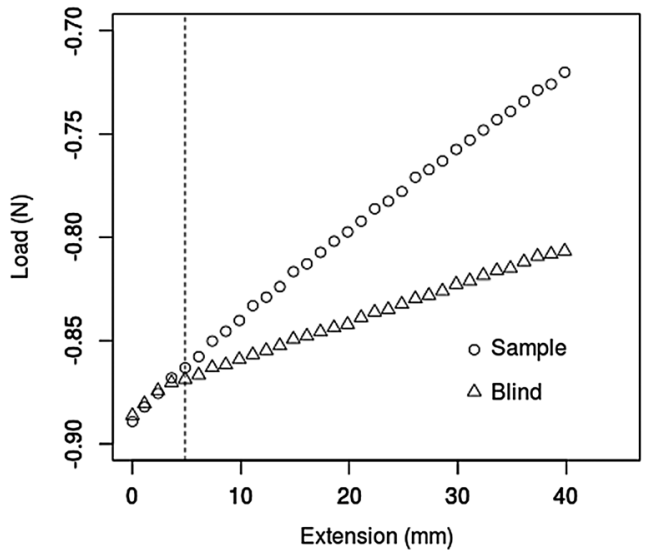

Fig. 13 Unidirectional extension of a hydrogel sample under distilled water (CD_400_1).

are all met. In this process small sample Monte Carlo simulations (50 samplings) were performed for a given sample $\left(G=500 \mathrm{~Pa}, h_{0}=100 \mathrm{~mm}, d_{0}=20 \mathrm{~mm}, N=10\right)$ under two different conditions (worst case:0 $c=10 \mathrm{~mm}$ per pix, $l(\mathrm{~mm})=10 \mathrm{~mm}$; best case: $c=100 \mathrm{~mm}$ per pix, $l(\mathrm{~mm})=$ $50 \mathrm{~mm}$ ). The results are presented in Fig. 14 and 15. From the two figures it is obvious that imprecise calibration produces strong exogeneity which damages homoscedasticity, resulting in potentially big errors in the calculated modulus and thus bad correlations between measured and simulated sample geometries.

The figures indicate that if one is capable of producing high resolution photographs, then linear regression is applicable, but otherwise other methods are required to determine the most probable modulus of the sample.

Non-linear regression is a convenient alternative as it takes the unsuspended distances as its input and applies the neo-Hookean law to the unsuspended distances in a certain modulus range, and finds the modulus, which produces the best fit. The result of such an approximation

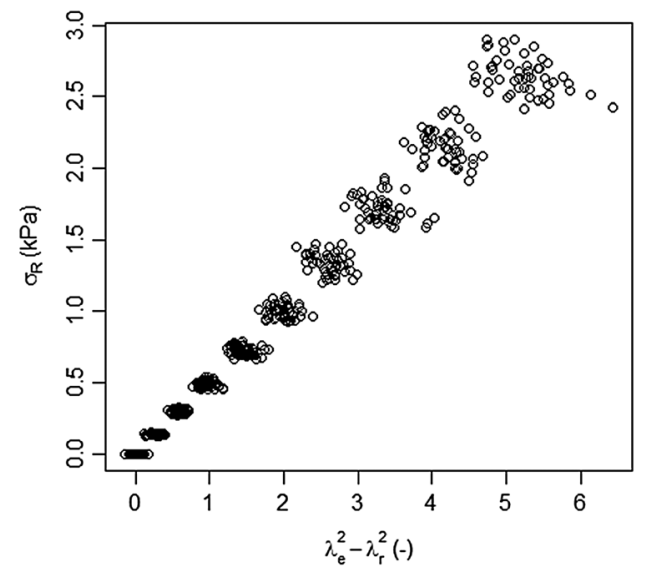

Fig. 14 Simulated measurements $(G=500 \mathrm{~Pa}, c=10$ pix per $\mathrm{mm}$, $\left.l(\mathrm{~mm})=10 \mathrm{~mm}, h_{0}=100 \mathrm{~mm}, d_{0}=20 \mathrm{~mm}, N=10\right)$.

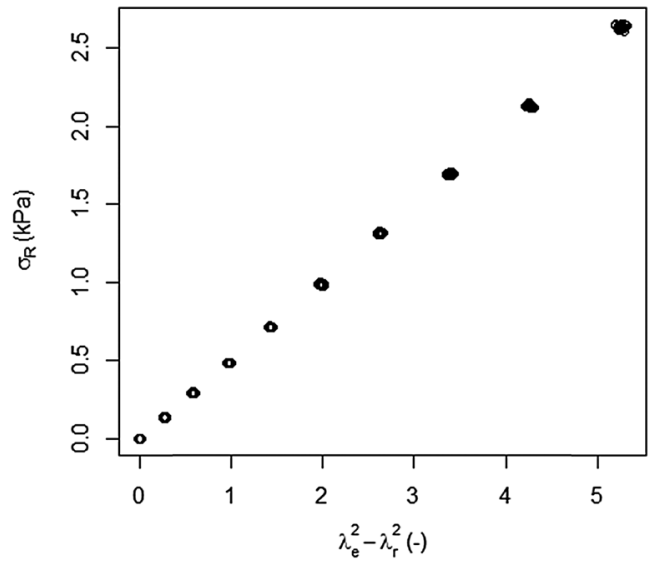

Fig. 15 Simulated measurements $(G=500 \mathrm{~Pa}, c=100$ pix per $\mathrm{mm}$, $\left.l(\mathrm{~mm})=50 \mathrm{~mm}, h_{0}=100 \mathrm{~mm}, d_{0}=20 \mathrm{~mm}, N=10\right)$.

for the sample previously presented in Fig. 8 is presented in Fig. 16. The figure shows that the actual modulus of the sample was lower than the one calculated by linear regression.

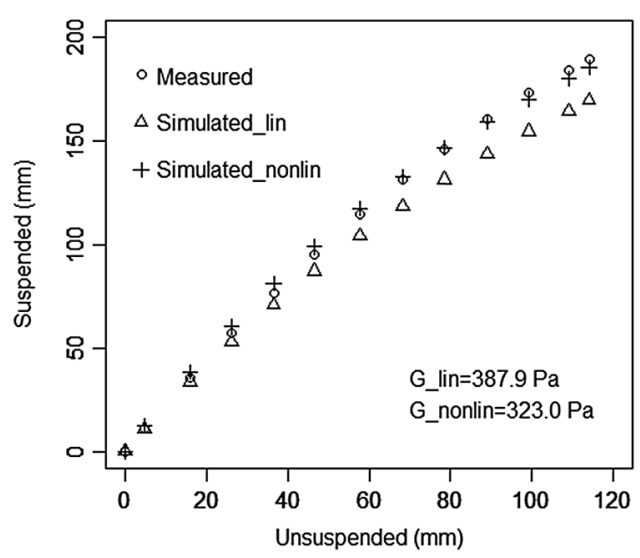

Fig. 16 Location of each mark on the unsuspended and suspended sample. Measurement and simulations. Simulations were run using moduli from both linear and non-linear approximations.

Table 3 Shear moduli estimated by robust linear and non-linear regression. RSS is the residual sum of squares between measured and simulated distances for both linear and nonlinear regression. Conv. is the shear modulus estimated from the results of conventional unidirectional stress-strain measurements performed in distilled water

\begin{tabular}{llllll}
\hline & $\begin{array}{l}G_{\text {R lin regr }} \\
(\mathrm{Pa})\end{array}$ & $\begin{array}{l}\mathrm{RSS} \\
\left(\mathrm{mm}^{2}\right)\end{array}$ & $\begin{array}{l}G_{\text {nonlin regr }} \\
(\mathrm{Pa})\end{array}$ & $\begin{array}{l}\mathrm{RSS} \\
\left(\mathrm{mm}^{2}\right)\end{array}$ & $\begin{array}{l}\text { Conv. } \\
(\mathrm{Pa})\end{array}$ \\
\hline CD300_1 & 749.2 & 82.313 & 1006.8 & 2.7240 & 863.8 \\
CD300_2 & 779.2 & 61.85 & 862.6 & 6.43 & 749.7 \\
CD300_3 & 621.6 & 48.50 & 680 & 8.03 & 614.6 \\
CD350_1 & 388.4 & 16.889 & 417.1 & 3.4993 & 462.1 \\
CD350_2 & 438.4 & 294.93 & 496.2 & 33.11 & 565.8 \\
CD350_3 & 439.6 & 938.75 & 581.6 & 17.22 & 616.6 \\
CD400_1 & 177.4 & 346.50 & 195.5 & 29.648 & 173.3 \\
CD400_2 & 362.4 & 171.86 & 365.5 & 169.36 & 343.9 \\
CD400_3 & 313.4 & 86.37 & 314.6 & 86.01 & 334.1
\end{tabular}




\section{Comparison with conventional unidirectional extension measurements}

It must be stressed that the "conventional" method is not conventional at all, due to its various limitations (e.g. difficulties in choosing a proper medium) described earlier. In our case however, distilled water proved to be a proper medium, therefore if the proposed method provided good results, the calculated moduli needed to be comparable with those determined by the conventional method. Results are presented in Table 3.

Table 3 shows that the results of the proposed new method and the so called "conventional" method differs by no more than approx. $20 \%$ which is acceptable, given the limitations of the conventional method.

\section{Conclusions}

In our work we have developed a new method to determine the modulus of extremely soft materials from their deformation under their own weight. The method is generally applicable to all kinds of soft materials irrespective of material composition with the restrictions that a cylindrical sample must be prepared from them and their deformation must be purely elastic and lack any form of plasticity. Test results with polyvinyl alcohol hydrogels show good correlation with those from underwater measurements which provide experimental proof for the applicability of the new method. The most important parameters affecting measurement uncertainty are the resolution of the photographs taken and the distances used for the calibration. The higher the resolution of the photograph and the calibration distances, the higher moduli the method can accurately determine, but for most commonly available imaging equipment, the measurement uncertainty due to pixel density constraints restricts the use of the method to moduli below $1000 \mathrm{~Pa}$. The accuracy of modulus determination can be further increased by substituting non-linear regression for linear regression.

\section{Notes and references}

1 X. L. Peng, J. Y. Huang, L. Qin, C. Y. Xiong and J. Fang, A method to determine Young's modulus of soft gels for cell adhesion, Acta Mech. Sin., 2009, 25(4), 565-570.

2 P. J. Flory, Principles of polymer chemistry. Ithaca, Cornell University Press, New York, 1967.

3 P. J. Flory and J. Rehner Jr, Statistical mechanics of cross-linked polymer networks II swelling, J. Chem. Phys., 1943, 11, 521-526.

4 K. Dusek and W. Prins, Structure and elasticity of noncrystalline polymer networks, Adv. Polym. Sci., 1969, 6, 1-102.

5 J. E. Mark and B. Erman, Rubberlike elasticity: a molecular primer, John Wiley \& Sons, New York, 1988.

6 F. Horkay and M. Zrinyi, Studies on mechanical and swelling behavior of polymer networks on the basis of the scaling concept. 6. gels immersed in polymer solutions, J. Macromol. Sci., Phys., 1986, B25(3), 307-334.

7 M. Zrinyi, H. G. Kilian and F. Horkay, On the uniaxial stress-strain dependence of highly swollen chemically crosslinked networks, Makromol. Chem., Macromol. Symp., 1989, 30, 199-214.

8 R. F. Boyer and R. L. Miller, Correlations involving the Mooney-Rivlin $C_{2}$ constant and the number of chain atoms between physical entanglements, $N_{\mathrm{c}}$, Polymer, 1987, 28, 399-407.

9 Joint Committee for Guides in Metrology: Evaluation of measurement data-Guide to the expression of uncertainty in measurement, JCGM 100:2008.

10 Joint Committee for Guides in Metrology: Evaluation of measurement data-Supplement 1 to the "Guide to the expression of uncertainty in measurement"-Propagation of distributions using a Monte Carlo method, JCGM 101:2008. 